\title{
Edge Detection using Third Order Difference Equation: A New Dimension
}

\author{
G.J.Joyce Mary, Ph.D \\ Associate Professor,Computer Science Engg. \\ PRIST University \\ Thanjavur,S.India
}

\author{
A.Rijuvana Begum \\ Research Scholar \\ PRIST University \\ Thanjavur,S.India
}

\begin{abstract}
Edges are the regions of object where there is a pointed change in intensity or gray level. Edge detection is one of the most frequently used operations in image analysis. If the edges are accurate, all the objects are located efficiently and the performance can be evaluated easily. Process of Edge detection is really a complicated task. Lot of research has been done in this field for many years. Object recognition, Target tracking, Segmentation, Data compression and Image matching are the most important areas of application where edge detection is highly required. This paper introduces a new edge detection technique using derivative mask. This mask is based on third order difference equation. The experimental results demonstrate the effectiveness of the new edge detector in terms of PSNR (Peak Signal to Noise Ratio) and MSE (Mean Square Error). Performance of this new mask compared with second order Laplacian Mask. Proposed mask is efficient and faster to implement. Experimental result on various images shows all the edges efficiently.
\end{abstract}

\section{Keywords}

Edge, Third Order, Difference equation, Mask, PSNR, MSE

\section{INTRODUCTION}

Human vision seems to make use of many sources of information to detect and recognize an object in a scene. At the lower level of object recognition, edge and region level of information are used to extract a 'perceptual unit' in the scene. [1] Edge is defined as the boundary pixels that connect two separate regions with changing image amplitude attributes such as different constant luminance and tristimulus values in an image[2] [3]. Edges include most useful information in an image. Edges provide important visual information and major physical, geometrical variations in scene object. Moreover an edge is a vector variable with two components magnitude and orientation, Edge magnitude (Edge strength) gives the amount of the difference between the pixels in the neighborhood, Edge orientation is the direction, it gives the direction of the greatest change [4].

Edge detection is an important image processing technique with wide range of applications. A number of edge detection techniques have been developed in the past few years, still no algorithm is completely suitable for all types of imaging conditions and applications [5]. Edge detection is the process that detects the presence and location of edges that arises due to sharp discontinuities in an image. The edge detection simplifies the image analysis process by considerably reducing the amount of data to be processed. Edge detection is difficult in noisy images, since both noise and edges are high frequency component [19]. There are five basic steps in edge detection, Smoothing, Filtering, Enhancement, Detection and
Localization [4]. This paper introduces a novel and efficient edge detection technique using the newly proposed third order difference equation. Proposed method provides better results for remote sensing images, medical images, forensic images, natural images, scanned real life images etc.

\section{RELATED WORK}

- In image mining applications human faces are detected using classical edged detectors. Performance analysis shows that the sobel is the best operator for face detection in terms of parameters like Accuracy, Speed and Confusion matrix which gives information on false positives, false negatives and true positives and true negative edges [5].

- Real life color images are analyzed using various edge detection algorithms like Prewitt, Sobel, Laplacian and Laplacian of Gaussian (LOG). Parameters PSNR, MSE and entropy are taken into account. Statistical results prove that Prewitt and Sobel gives clear edge detection performance than Laplacian and LOG [6].

- $\quad$ SHARK fish is classified based on Roberts, Prewitt, Sobel, Laplacian and Canny filters. It is proved that the Canny produces good adaptability in filter designing to suit for the different noisy environments at the expense of processing speed and cost [7]

- A new method of edge detection framed based on binary morphology and border line closed technique used to extract image edges [8].

- Optical character, Finger print and Iris are recognized using Canny and Gabor edge detectors. Results prove that the Gabor is a linear filter that represents frequency and orientations which are similar to human visual system and it directly uses Gabor wavelet which are generated from Mother wavelets called Dilation and Erosion gives good edge map [9].

- Car license plates are detected using a new approach called Vertical Edge Detection Algorithm (VDEA) and Structured Component algorithm for its accurate identification [10].

- An innovative approach for detecting fine edges in an image based on morphological operators whose Structured Element dynamically varies with the Chain code. Real life and synthetic images are experimented. The proposed method outperforms 
the canny operator that helps to remove noise contained in an image [11].

- Sobel edge mask of size 5x5 was developed by using directional derivative approach. The classical sobel operator is of the size $3 \times 3$. This new $5 \times 5$ mask produces better performance of edge detection at the expense of execution speed than the classical sobel mask [12].

- A new method of edge detection using additions and divisions proposed in which threshold value is automatically computed for the efficient detection of edges [13].

\section{EDGE DETECTION TECHNIQUES}

There are so many methods to perform edge detection. Edge detection uses differential operator to determine the changes in the gray level. The process of edge detection is basically divided into two main categories, Gradient and Laplacian. Gradient methods detect the edges by finding for the maximum and minimum in the first order derivative of the image. The Laplacian method is based on zero crossing for the second order derivative of an image to detect and locate the edges. This section briefs Gradient operators Robert, Prewitt and Sobel and also second order Laplacian operator.

\subsection{First Order Edge Detection}

It is otherwise called as gradient based method. It makes use of first order derivative of an image to determine its gradient. The gradient of a two dimensional image function $f(i, j)$ is a vector and given by following expression[2].

$$
\nabla f=\partial f / \partial i+\partial f / \partial j-----(1)
$$

\subsubsection{Robert Operator}

It uses two masks of size $2 \times 2$ one for each direction to compute the sum of the squares of the difference between diagonally adjacent pixels through discrete differentiation and then calculate approximate gradient of the image. The input image is convolved with the default kernels of operator and gradient magnitude and directions are computed. The masks are designed to produce maximal response at 45 degree to the pixel grid [15].

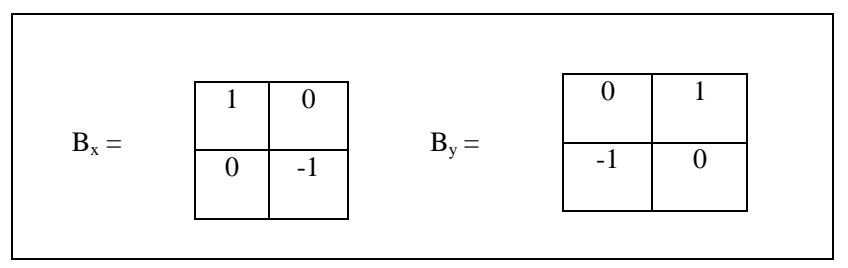

\section{Fig.1.Roberts Mask}

Gradient magnitude is given by:

$$
|B|=\sqrt{B_{x}^{2}}+B_{y}^{2}
$$

The angle of orientation of edge relative to the pixel grid is given by:

$$
\Theta_{B}=\arctan \left(B_{y} / B_{x}\right)-3 \pi / 4
$$

Since the masks are of smaller in size, it is very simple to handle, but sensitive to noise and less compatible with recent technology.

\subsubsection{Prewittt Operator}

Robert mask is uncomfortable to use since it do not have clear centre pixel. Hence it is enlarged to $3 \times 3$ size which is Prewitt mask. It reduces the influences of the noise while detecting true edges. It computes the gradient of the image surrounded by the $3 \times 3$ mask. These masks shown below are then convolved with the image to obtain the derivatives of the horizontal and vertical edges separately. Due to large mask size it has low computational ability but less sensitive to noise [13][17].

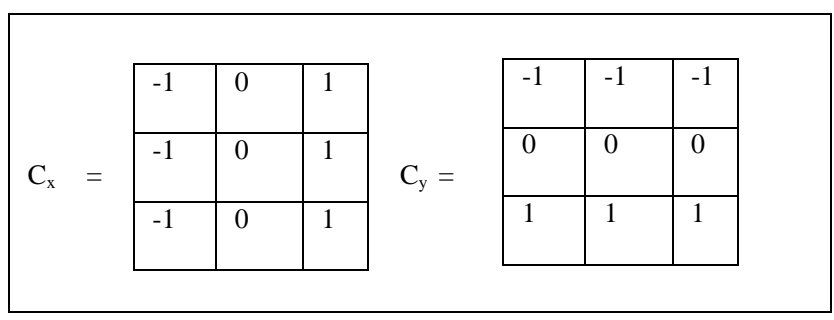

Gradient magnitude:

Fig.2.Prewitt Mask

$$
|C|=\sqrt{ } C_{x}^{2}+C_{y}^{2}
$$

The angle of orientation of edge:

$$
\Theta_{C}=\arctan \left(C_{y} / C_{x}\right)
$$

\subsubsection{Sobel Operator}

It is a modified Prewitt operator by inserting a scale factor of 2 at the centre indicating that it is more emphasized. At each pixel of an image, sobel operator gives either the corresponding gradient vector or normal to the vector. It computes the gradient magnitude and the direction by convolving image with the following two masks,

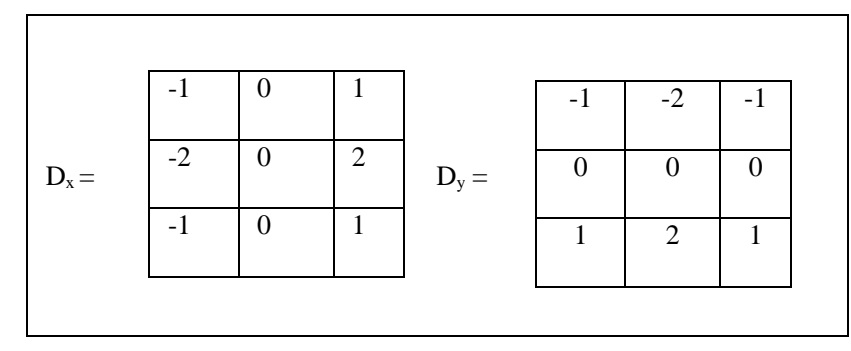

Gradient magnitude:

Fig.3.Sobel Mask

$$
|D|=\sqrt{ } D_{x}^{2}+D_{y}^{2}
$$

The angle of orientation of edge:

$$
\Theta_{D}=\arctan \left(D_{y} / D_{x}\right)
$$

This operator has good noise suppression capability than Prewitt mask [15].

\subsection{Second Order Edge Detection}

It is otherwise called as Laplacian based edge detector uses a second order partial derivative. Its zero crossing property detects whether the pixel belongs to either dark or bright side of the gray scale.

The Laplacian of a two dimensional image function $f(i, j)$ is a second order derivative [2] which given by, 


$\nabla^{2} f=\partial^{2} f / \partial i^{2}+\partial^{2} f / \partial j^{2}-$
$G=\quad$\begin{tabular}{|c|c|c|}
\hline 0 & -1 & 0 \\
\hline-1 & 4 & -1 \\
\hline 0 & -1 & 0 \\
\hline
\end{tabular}

\section{Fig.4.Laplacian Mask}

The convolution mask of the Laplacian operator of size $3 \times 3$ is shown in fig.4. This mask is isotropic (Rotation invariant) [14].

\section{PROPOSED METHOD}

The Robert first order derivative operator of size $2 \times 2$ approximates the gradient of an image by discrete differentiation. Discrete differentiation is done by computing sum of the squares of differences between diagonally adjacent pixels. It quickly compute 2D spatial gradient of input image at that point. But it fails to detect true edges because of its small mask size. Also it is highly sensitive to noise. Further the operators Prewitt and Sobel of enlarged size $3 \times 3$ works on convolution of image with small separable and integer valued filter in both horizontal and vertical directions. For high frequency, it produces crude gradient. It also proves that it is easy to handle images since it has clear centre pixel. Though these operators are less sensitive to noise, they have a tendency to produce thick edges [17]. Besides, all the first order operator uses two masks one for each direction. Second order Laplacian operator involves a single $3 \times 3$ isotropic mask which produces thinner edges near the object boundaries. Its zero crossing property detects the location of the edges too. To improve the edge detection performance further, a new method has been proposed using third order difference equation. It produces a single mask of size $4 \times 4$ which directly operated on the image pixels to determine the edge map. The newly proposed mask is applied to different images showing good detection ability interms of high PSNR and low MSE values. Formulation of newly proposed mask as follows.

With reference to the equation (2), third order difference equation is written as

$$
\nabla^{3} f=\partial^{3} f / \partial i^{3}+\partial^{3} f / \partial j^{3}-----(3)
$$

Image points of high contrast can be detected by computing intensity differences in local image regions. Typically, such points form the border between different objects or scene parts. With the help of one-dimensional signals the difference equation and hence the mask is developed. Since the 1D signals could just be rows or columns of a 2D image [18]. Considering a one-dimensional continuous function $\mathrm{f}(\mathrm{i})$, the third order difference equations are derived in steps of first and second order as shown in fig.5. $\mathrm{f}^{\prime}(\mathrm{i}), \mathrm{f}^{\prime \prime}$ (i) and $\mathrm{f}^{\prime \prime}$ (i) are the first, second and third differences of $\mathrm{f}(\mathrm{i})$.

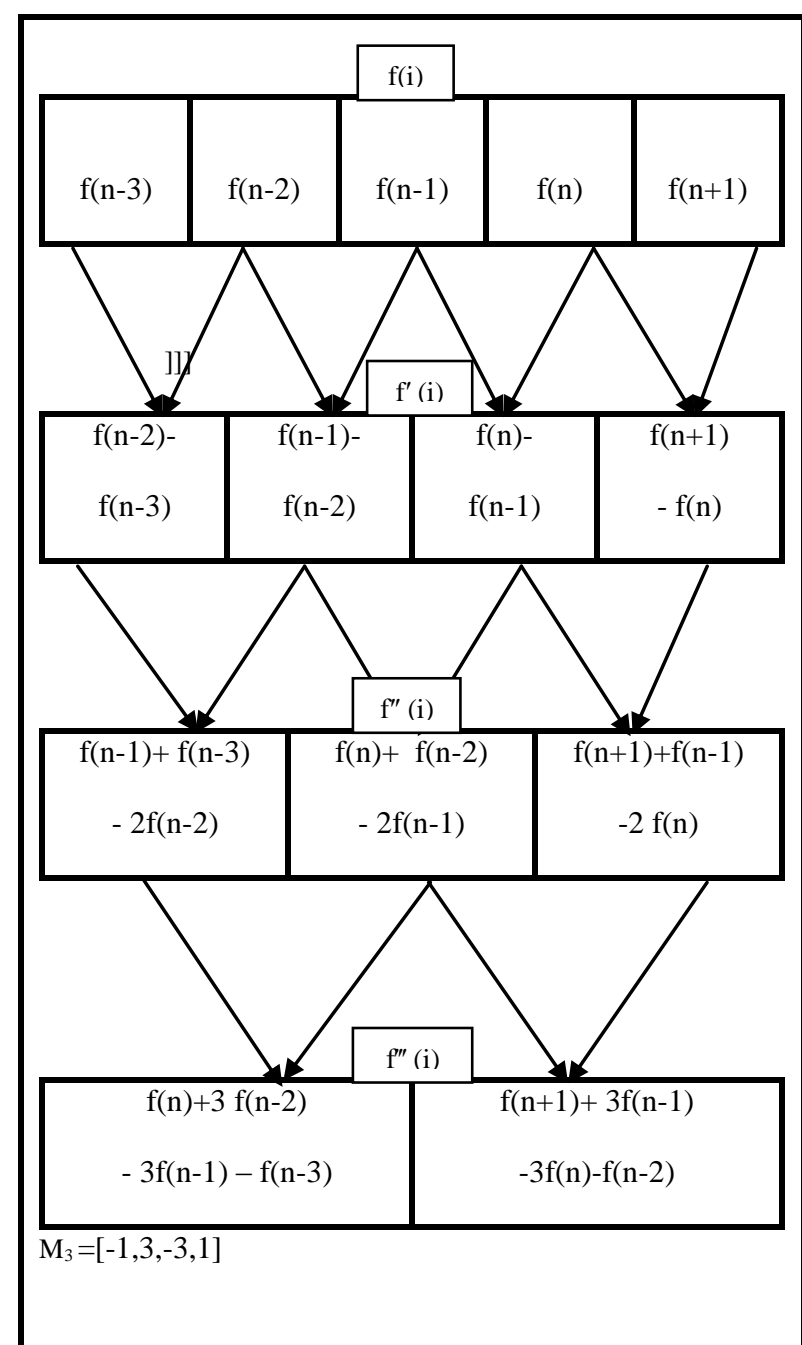

Fig.5.Discrete samples of $f(i), 1^{\text {st }}$ difference, $2^{\text {nd }}$ difference, $3^{\text {rd }}$ difference

From the $3^{\text {rd }}$ order one-dimensional mask $\mathrm{M}_{3}$ two dimensional mask of size $4 \times 4$ is developed as shown in fig. 6 .

$\mathrm{E}=\quad$\begin{tabular}{|c|c|c|c|}
\hline 0 & 0 & -1 & 0 \\
\hline 0 & 0 & 3 & 0 \\
\hline-1 & 3 & -6 & 1 \\
\hline 0 & 0 & 1 & 0 \\
\hline
\end{tabular}

Fig.6.Proposed Mask

By convolving this mask over the image, an edge map has been obtained for the images.

\section{EXPERIMENTAL RESULTS}

To analyze the performance of the proposed mask it is implemented in MATLAB software. Forensic fingerprint image, MRI brain image, binary image and natural images of different sizes, various formats is tested using second order 


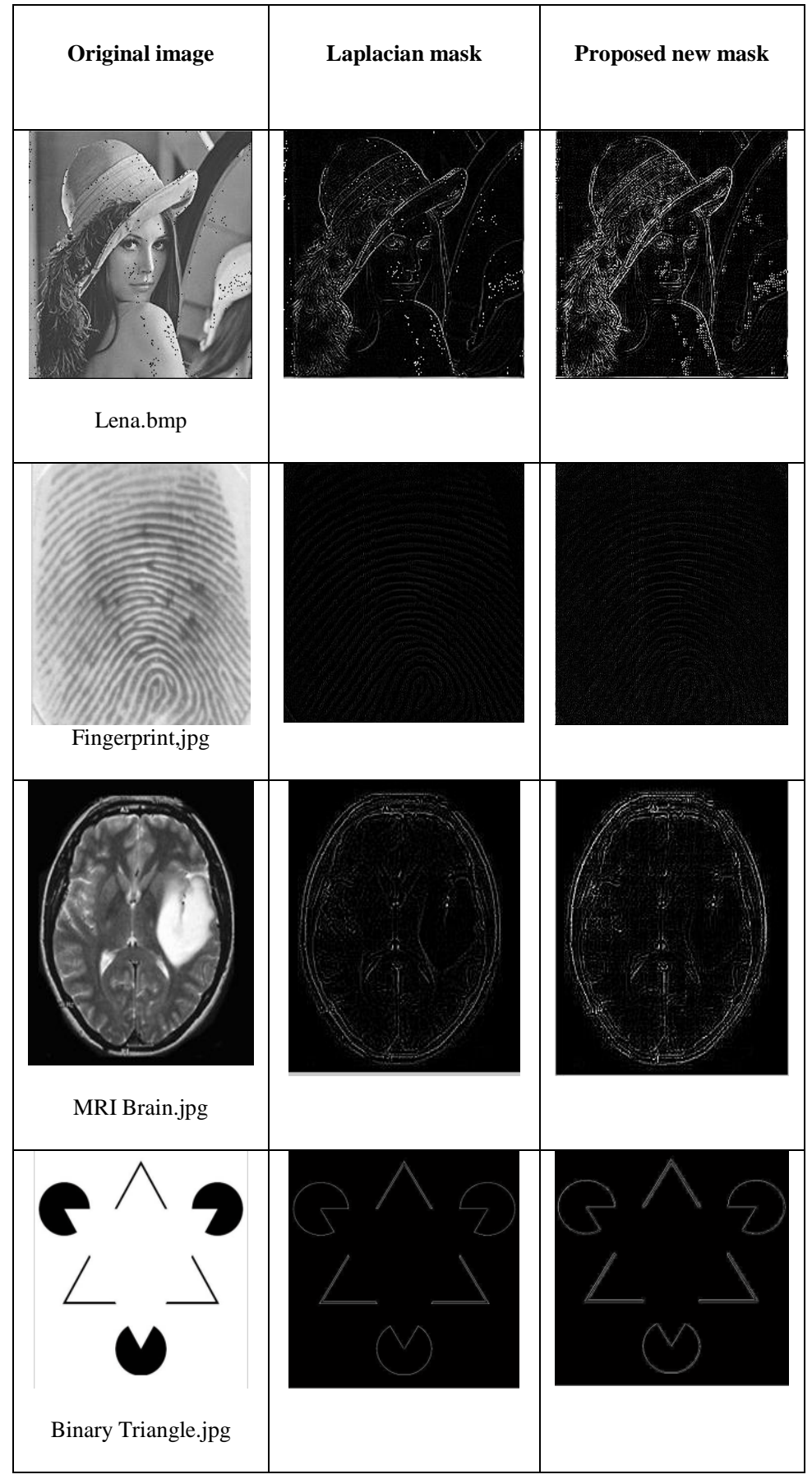

Fig.7. Resultant Images after applying edge detection mask

Laplacian and newly proposed third order mask. Results shown in fig.7 states that the proposed method gives better edge map than Laplacian based edge detection algorithm.

Table.1 shows the comparative analysis of classical second order Laplacian edge detection algorithm with newly proposed technique. Parameters such as Peak Signal to Noise Ratio (PSNR) and Mean Square Error (MSE) are taken for consideration. Variety of images and different file formats and sizes are taken into account. For an example a bitmap lena noisy image of size 209 × 209 has been processed using both Laplacian and newly proposed mask. PSNR of Laplacian operated image is 8.3646 , where as the proposed new mask provides higher PSNR of 8.993. Also MSE Value of Laplacian method is $9.472 \times 10^{3}$, but the newly proposed mask offer smaller value such as $8.199 \times 10^{3}$. Likewise for the other images also the proposed new mask produces high PSNR and Low MSE compared to Laplacian operator.

Table 1.Performance Comparison

\begin{tabular}{|c|c|c|c|c|}
\hline \multirow{2}{*}{$\begin{array}{c}\text { Sample } \\
\text { Images }\end{array}$} & \multicolumn{2}{|c|}{ Laplacian Mask } & \multicolumn{2}{c|}{ Proposed mask } \\
\cline { 2 - 5 } & $M S E$ & $P S N R$ & $M S E$ & PSNR \\
\hline Lena & $9.472 \times 10^{3}$ & 8.3646 & $8.199 \times 10^{3}$ & 8.993 \\
\hline Fingerprint & $1.812 \times 10^{4}$ & 5.5472 & $1.772 \times 10^{4}$ & 5.644 \\
\hline MRI brain & $4.860 \times 10^{3}$ & 11.260 & $4.522 \times 10^{3}$ & 11.57 \\
\hline Binary image & $4.134 \times 10^{4}$ & 1.9064 & $4.051 \times 10^{4}$ & 2.0546 \\
\hline
\end{tabular}

Comparative performance is also depicted graphically in fig. 8 and fig.9.

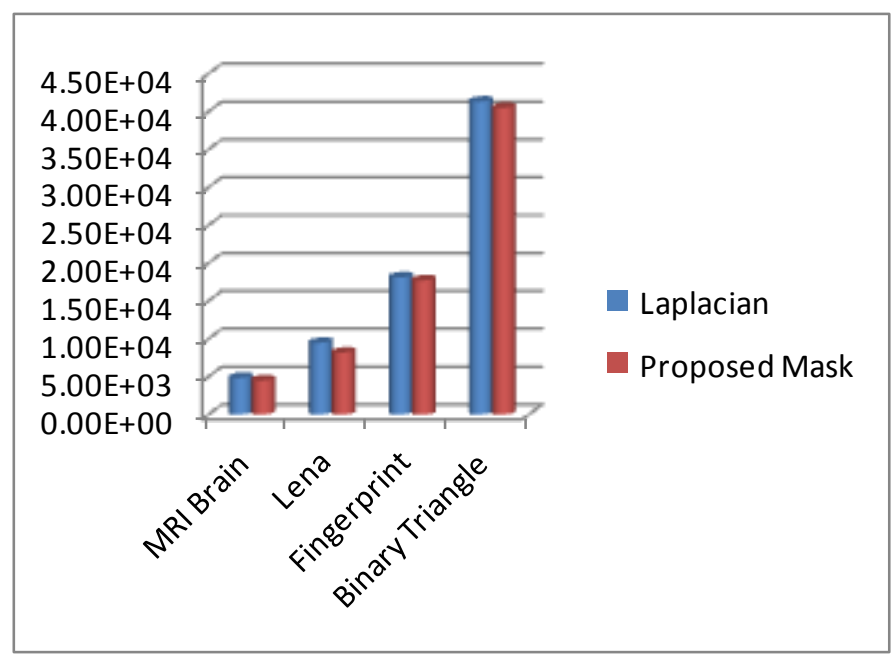

Fig. 8.Mean Square Error (MSE)

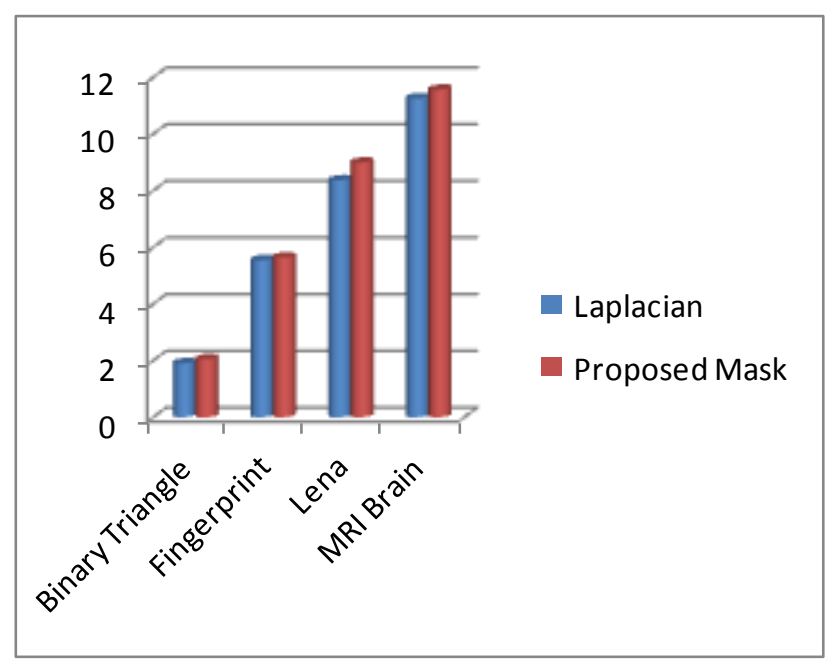

Fig. 9.Peak Signal to Noise Ratio (PSNR) 
Mean Square Error (MSE) graphically represented in fig. 8 shows the comparison between existing second order Laplacian with newly proposed third order derivative mask. It is observed that the proposed new mask provides smaller MSE values for all the example images taken for consideration. Also in fig.9 Peak signal to Noise Ratio (PSNR) is represented. It shows the proposed new mask outperform by providing higher values than the existing Laplacian mask.

\section{CONCLUSION AND FUTURE WORK}

Edge detection is a fundamental step for image segmentation used for object detection and many other applications. Therefore it is necessary to use a strong edge detector which gives better results at all situations. In this paper it has been proposed that a new edge detector based on third order difference equation. First order Robert mask is one of the oldest method and it is used where simplicity and speed are dominant factors, but it is highly sensitive to noise. On the other hand Prewitt and Sobel masks are less sensitive to noise but produces thicker edges. So the edge localization is poor. Second order Laplacian mask is a single isotropic mask which offers thin edges and good localization. Proposed new mask tested on various images proves better results than second order Laplacian mask in terms of low MSE and high PSNR values. Newly proposed mask using third order difference equation is an enhancer for edge detection. To extract all the edges sharply in noisy environments, a suitable smoothing noise reduction and edge preserving technique is used in future. Also by incorporating possible improvements, newly proposed technique can directly applied to the color images without converting them into grayscale images.

\section{REFERENCES}

[1] Hankyu Moon, Rama Chellappa, Azreil Rosemfeld 2002,"Optimal edge based shape detection", IEEE Trans. on Image Processing ,vol .11,No.11,pp.1209-1226.

[2] Gonzalez,R.C.Woods,R.E,2001 Digital Image Processing. Prentice Hall, $3^{\text {rd }}$ ed.

[3] William. Pratt.K, 1991 Digital Image Processing WillyInterscience.

[4] Jayakumar,R. Suresh,B 2014 "A review on edge detection methods and techniques" IJARCCE,Vol.3,Issue 4.

[5] Vijayarani,S.Vinupriya,M.2013 "Performance analysis of canny and sobel edge detection algorithms in image mining “,IJIRCCE,Vol.1,Issue 8, pp-1760-1767.

[6] Pinaki Pratim Acharjya, Ritaban Das, Dibyendu Ghoshal 2012“ A study onimage edge detection using the gradients", International Journal of Scientific and Research Publications, Vol. 2, Issue 12.
[7] Shrivakshan,G.T, Dr. Chandrasekar,C 2012“ A comparison of various edge detection techniques used in image processing "International Journal of Computer Science Issues, Vol. 9, Issue 5, No 1, pp-269-276.

[8] Kalpana,M, Kishorebabu,G, Sujatha,K 2012 "Extraction of edge detection using digital image processing techniques" International Journal Of Computational Engineering Research , Vol. 2, Issue.5, pp-1562-1566.

[9] Masoud Nosrati ,Ronak Karimi Mehdi Hariri Kamran Malekian 2013“Edge detection techniques in processing digital images:Investigation of canny algorithm and gabor method" World Applied Programming Journal, Vol. 3, Issue 3, pp.116- 121.

[10] Siva Vinayaka Pavana Hiranya ,P, Venkata Suresh, B , Sabhanayagam, T 2014 "Car license plate detection using structured component analysis "IJEDR, pp-196200.

[11] Ratika Pradhan, Prasanna Pradhan, Reshmi Bhattacharjee, Divyendra Singh, "Edge detection using morphological operator: A new approach" International Journal of Advanced Research in omputer Science and Software Engineering,vol.4,issue.2,pp-84- 87,Feb.2014.

[12] Elif Aybar , "Sobel edge detection method for MATLAB“

[13] Sabina Priyadarshini, Gadadhar Sahoo, 2010“A New Edge DetectionMethod based on Additions and Divisions" International Journal of Computer Applications, Vol. 9.No.10,pp.10-13.

[14] Rashmi,Mukesh Kumar, Rohini Saxena, 2013 "Algorithm and technique on various edge detection: a survey“ SIPIJ,Vol.4, No.3, pp-65-75.

[15] Kiranjeet Kaur,Sheenam Malhothra, 2013“A survey on edge detection using different techniques“,IJAIEM,Vol.2,Issue.4,pp-496-500.

[16] Chinu,Amit Chhabra, 2014"Overview and comparative analysis of edge detection techniques in digital image processing",International Journal of Information and Computer Technology,Vol.4,No.10,pp.973-980.

[17] Ashita Vermani, Akshyata Ojha, 2015“ Algorithms for edge detection and enhancement for real time images: A comparative study "IJIRCE,Vol. 3, Issue 2,pp-651-658.

[18] Shapiro and Stockman,2000, Computer Vision.

[19] Raman Maini,Dr.Himansu Agarwal, "Study and comparison of various image edge detection techniques", IJIPVol.3,Issue.1. 